\title{
Gut microbes in healthy dogs have individualized responses to synbiotic supplementation that lead to increased abundances of probiotic strains: $A$ randomized controlled trial
}

Jirayu Tanprasertsuk

NomNomNow https://orcid.org/0000-0001-6203-3797

Aashish R Jha

New York University Abu Dhabi

Justin Shmalberg

University of Florida

LeeAnn M Perry

NomNomNow

Heather Maughan

Ronin Institute: Ronin Institute for Independent Scholarship

Ryan W Honaker ( $\square$ ryan.honaker@nomnomnow.com )

University of Florida https://orcid.org/0000-0003-3459-628X

\section{Research Article}

Keywords: diarrhea, dogs, gastrointestinal health, gut microbiome, metagenomic sequencing, probiotics, synbiotics

Posted Date: November 5th, 2020

DOl: https://doi.org/10.21203/rs.3.rs-100335/v1

License: (c) (1) This work is licensed under a Creative Commons Attribution 4.0 International License. Read Full License 


\section{Abstract}

Background: Probiotics ameliorate gastrointestinal symptoms in dogs. However, the effect of probiotics in a healthy population, as well as factors contributing individualized responses, remains largely unknown. This trial examined gut microbiota (GM) and health outcomes in household dogs after synbiotic (SN) supplementation containing probiotics and inulin. Healthy dogs were randomized to receive $S N(50 \mathrm{mg} / \mathrm{d}$ inulin and 20 billion total $\mathrm{CFU} / \mathrm{d}$ of L. reuteri, P. acidilactici, E. faecium, L. acidophilus, B. animalis, L. fermentum, L. rhamnosus) or placebo (PL) for 4 weeks. Owners completed a health survey and collected stool samples for GM profiling (metagenomic sequencing) at baseline and week 4 in both groups, and at week 6 in the SN group.

Results: A significant shift $(\mathrm{p}<0.001)$ in $\beta$-diversity was observed in the $S N(n=24)$, but not PL group $(n=19)$, relative to baseline at week 4 . Forty-five bacterial species, $43(96 \%)$ of which were Lactobacillales, showed an increase in the abundances ( $\geq 2$ fold change, adjusted $p<0.05$ ) at week 4 . E. coli also decreased at week 4 in the SN group ( 2.8 folds, adjusted $p<0.01$ ). The altered taxa largely returned to baseline at week 6 . The degree of changes in the $\beta$-diversity was associated with $\mathrm{GM}$ at baseline. Specifically, dogs with higher Proteobacteria and lower Lactobacillales responded more robustly to supplementation. Dogs fed SN tended to have lower diarrhea incidence ( $0 \%$ vs $16 \%, p=0.08)$.

Conclusions: SN supplement had an impact on the change of gut microbiota in healthy household dogs as characterized with metagenomic sequencing. Findings warrant further investigation with longer duration or in populations at risk of gastrointestinal diseases. The magnitude of response to the supplement was associated with microbial profile at baseline. To our knowledge, this is the first study documenting such association that may provide a basis of personalized nutrition in companion dogs.

\section{Full Text}

This preprint is available for download as a PDF.

\section{Tables}

Due to technical limitations, table 1 to 6 is only available as a download in the Supplemental Files section.

\section{Figures}




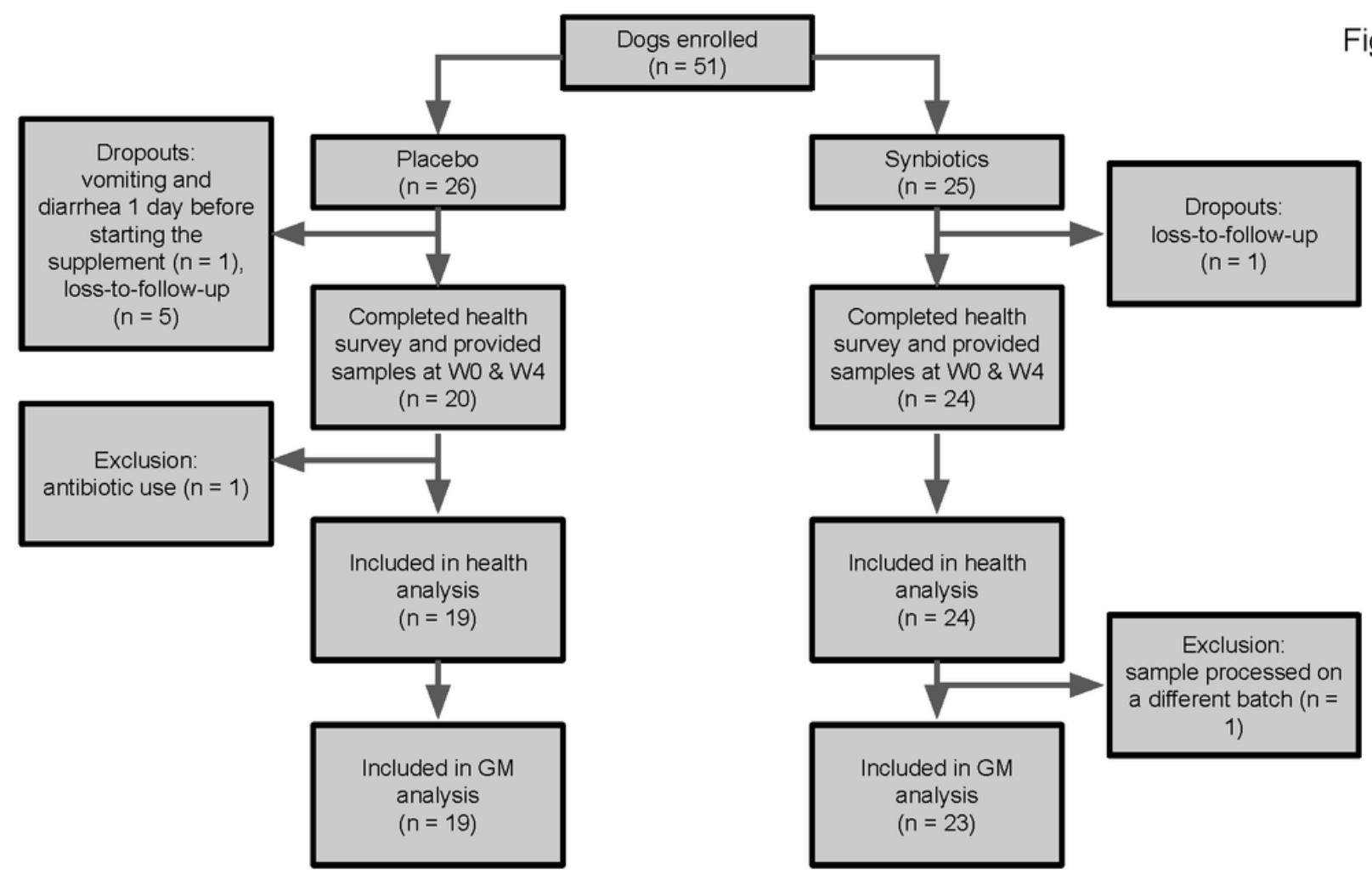

Figure 1

Trial flowchart
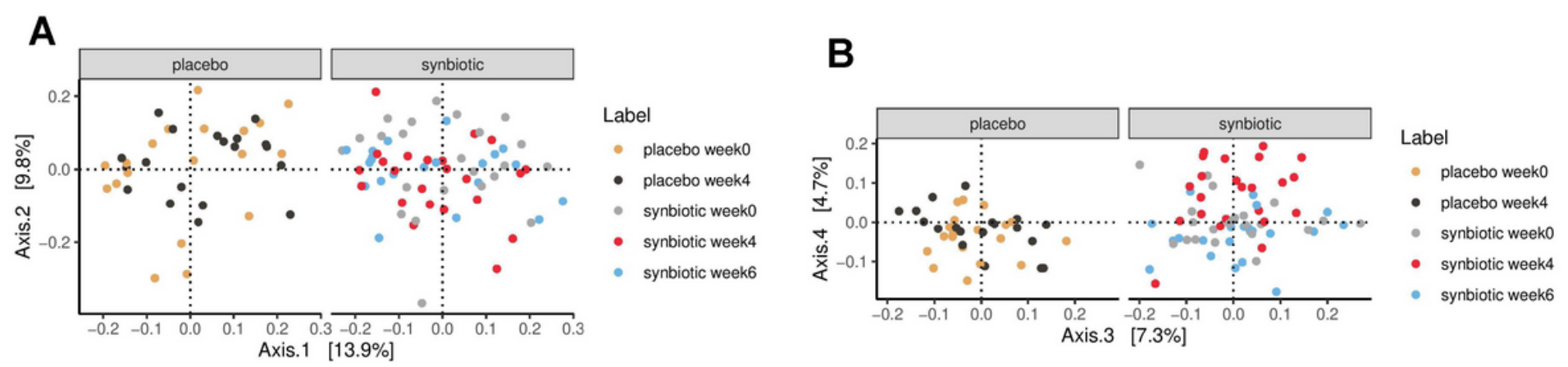

Figure 2

Principal coordinate analysis (PCoA) plot. (A) PCoA1 (Axis 1) and PCoA2 (Axis 2) respectively explained $13.9 \%$ and $9.8 \%$ of the variance of the abundance of gut microbiota at the species level (105 samples from 42 dogs). PERMANOVA using Bray-Curtis distance showed no spatial separation among treatment groups (placebo and probiotics) or timepoints (weeks $0,4,6$ ) based on PCoA1 and PCoA2 scores. (B) PCoA1 and PCoA4 (explaining 4.7\%). 

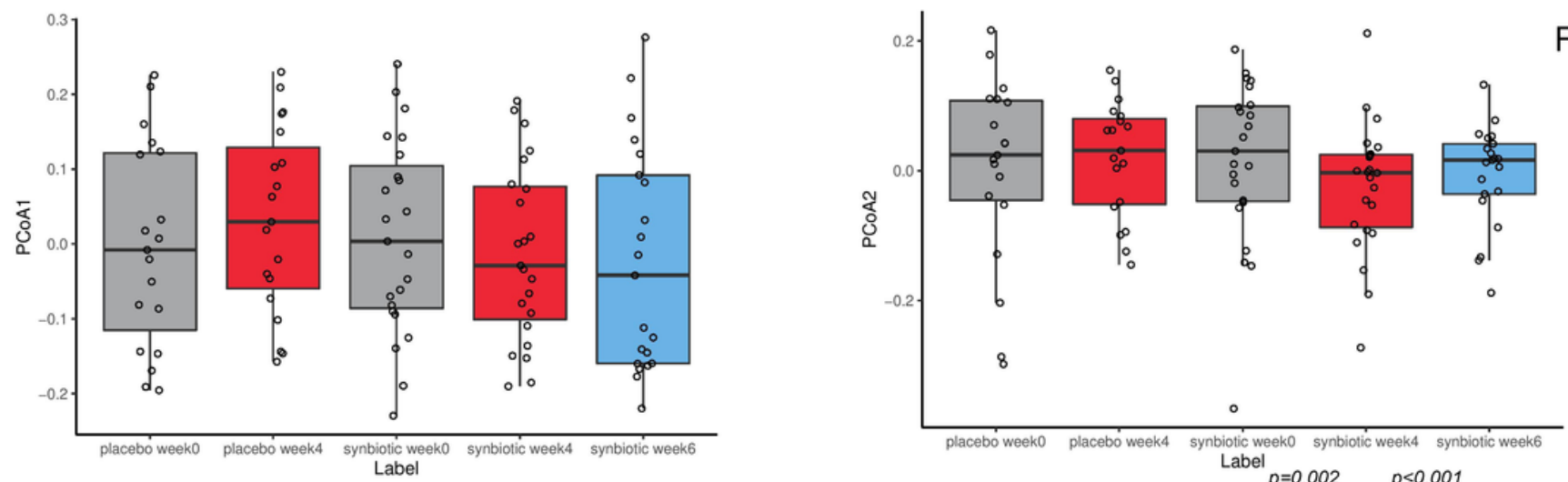

Fig 3
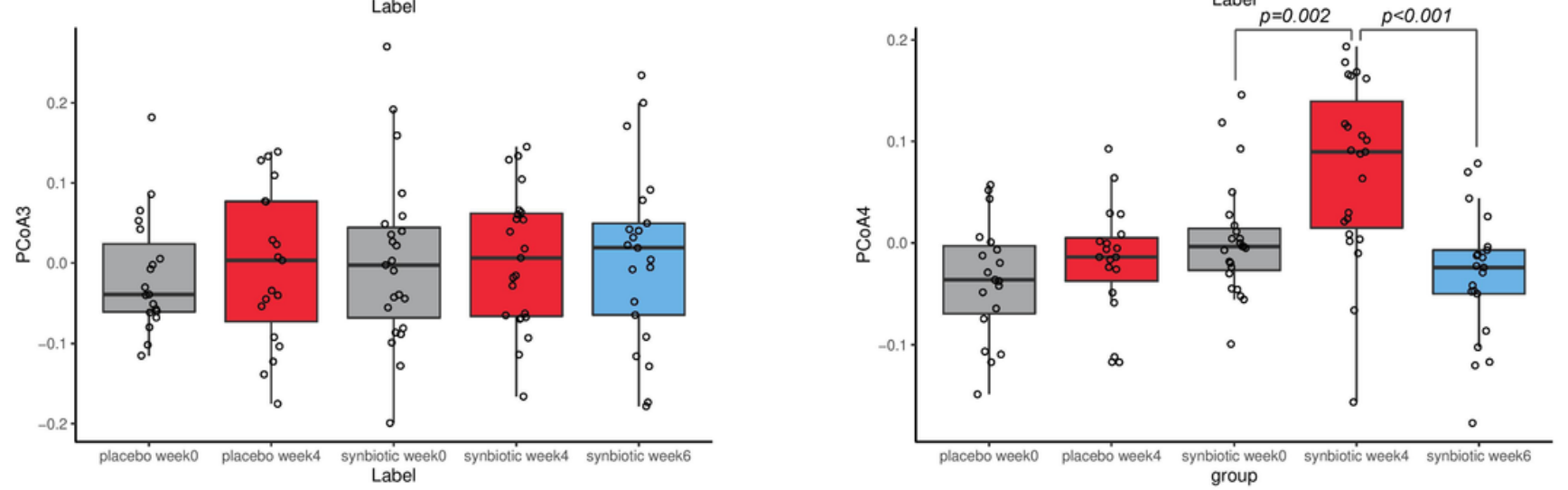

Figure 3

Scores of the first 4 PCoA axes in subjects receiving synbiotic $(S N, n=23)$ or placebo $(P L, n=19)$ at weeks 0,4 , and 6 . PCoA4 score in the synbiotic group at week 4 was significantly different from that at week 0 (adjusted $p=0.002$ ) and week 6 (adjusted $p<0.001$ ). P value adjustment for pairwise comparisons was performed using false discovery rate. 
A

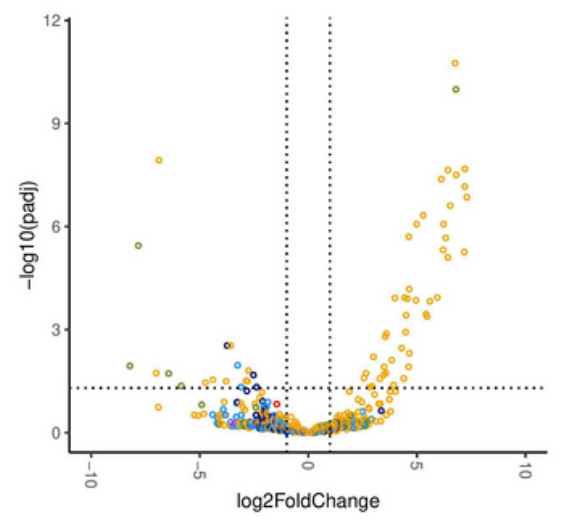

C

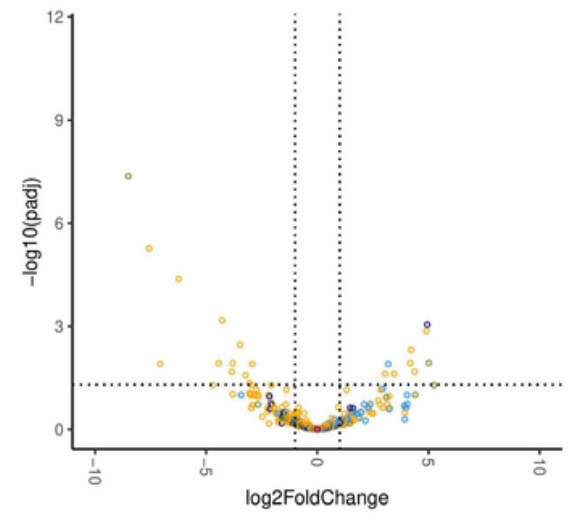

Rank2

- p_

- p_Actinobacteria

- p_Bacteroidetes

- P_Firmicutes

- P_Fusobacteria

- P_Proteobacteria

- P_Tenericutes

- P_Verrucomicrobia

Rank2

P_

- P_Actinobacteria

- P_Bacteroidetes

P_Firmicutes

- P_Fusobacteria

P_Proteobacteria
B

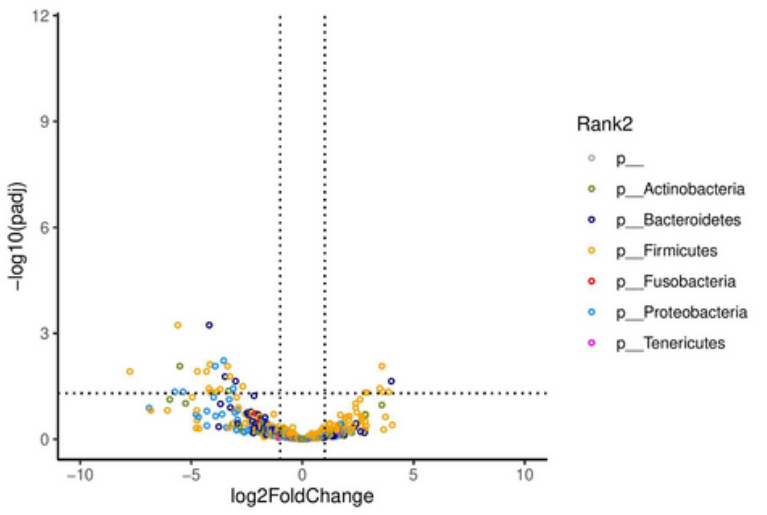

Fig 4

\section{Figure 4}

Volcano plots demonstrating the fold-change (FC) in the abundance of gut bacteria at the species level (A) at week 4 compared to week 0 in the synbiotic group $(n=23)$; (B) at week 6 compared to week 0 in the synbiotic group ( $n=21)$; and (C) at week 4 compared to week 0 in the placebo group $(n=19)$. Vertical dashed lines show log 2 FC at 1 and -1 (i.e. FC at 2 and -2). Horizontal dashed line shows - $\log 10$ $($ adjusted $p)=2$ (i.e. adjusted $p=0.01$ ). Points are colored by phylum . 

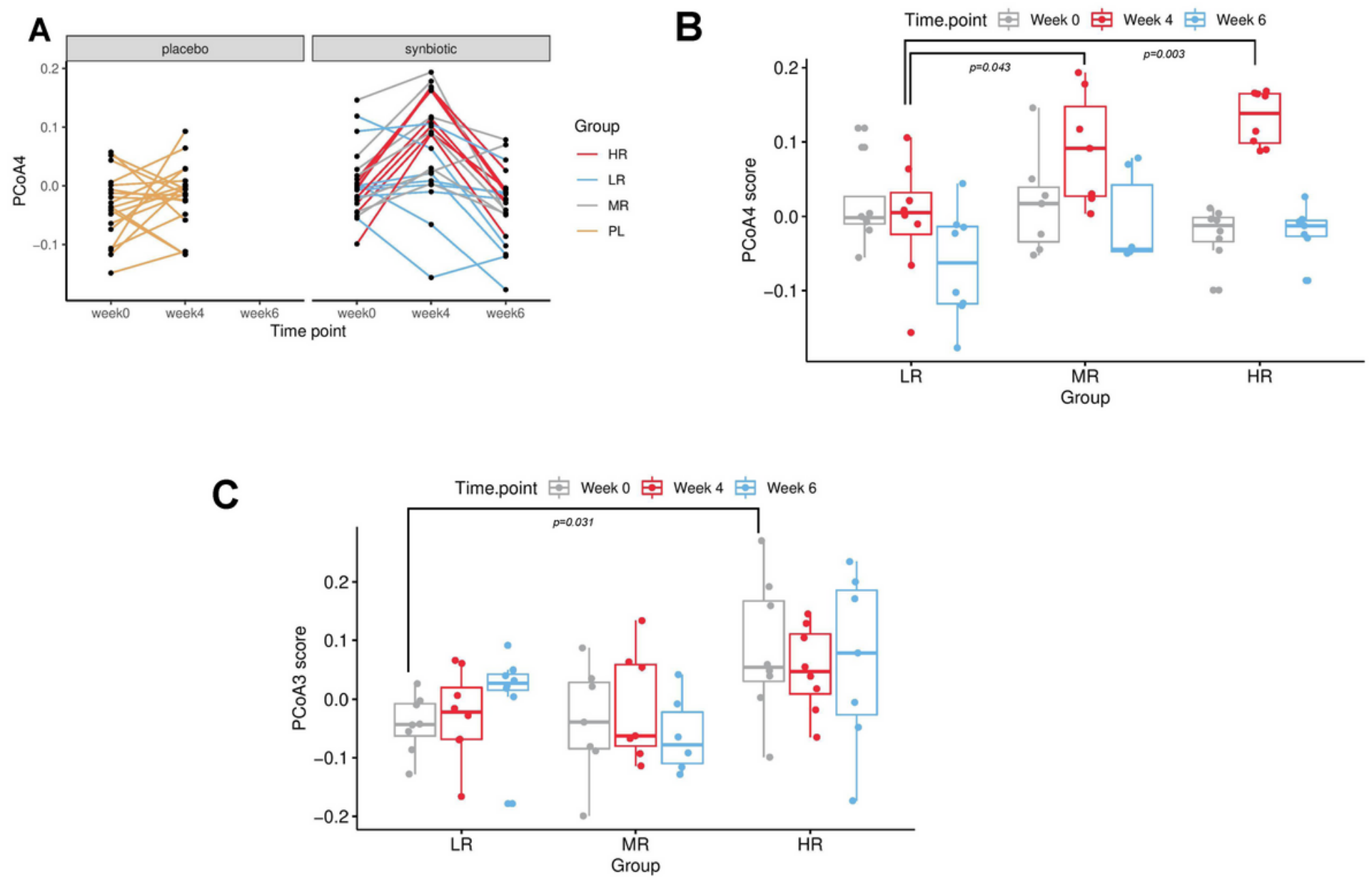

\section{Figure 5}

Varying degrees of PCoA4 changes from week 0 to week 4 were observed among subjects receiving the synbiotic supplement. (A) PCoA4 score at week 4 increased in 20 dogs (87\%) as compared to baseline in the synbiotic group, while the direction of change was less consistent in the placebo (PL) group increased in 11 dogs (58\%) and decreased in 8 dogs (42\%). Subjects in the synbiotic group were divided into tertiles based on the degree of PCoA4 changes between week 0 and week 4, with subjects in the first tertile labeled high-responders (HR, maximal PCoA4 score increase, $n=8)$, those in the second tertile midresponders $(M R, n=7)$, and those in the third tertile low-responders ( $L R, P C O A 4$ score decrease or minimal PCoA4 score increase, $n=8)$. (B) PCoA4 scores at week 4 were significantly higher in HR $(0.132 \pm 0.037$, FDR-adjusted $p=0.003$, pairwise Wilcoxon rank sum test $)$ and MR $(0.091 \pm 0.076$, FDR-adjusted $p=$ $0.043)$ as compared to LR $(-0.004 \pm 0.080)$. (C) PCoA3 scores were significantly higher in HR $(0.084 \pm 0.117)$ than LR $(-0.043 \pm 0.049)$ but not MR $(-0.038 \pm 0.096)$ at baseline (FDR-adjusted $p=0.031$, Kruskal-Wallis test). PCoA3 scores were not significantly different among groups at week 4 or week 6 . Values sharing the same superscript are not statistically significant from each other. 
A B
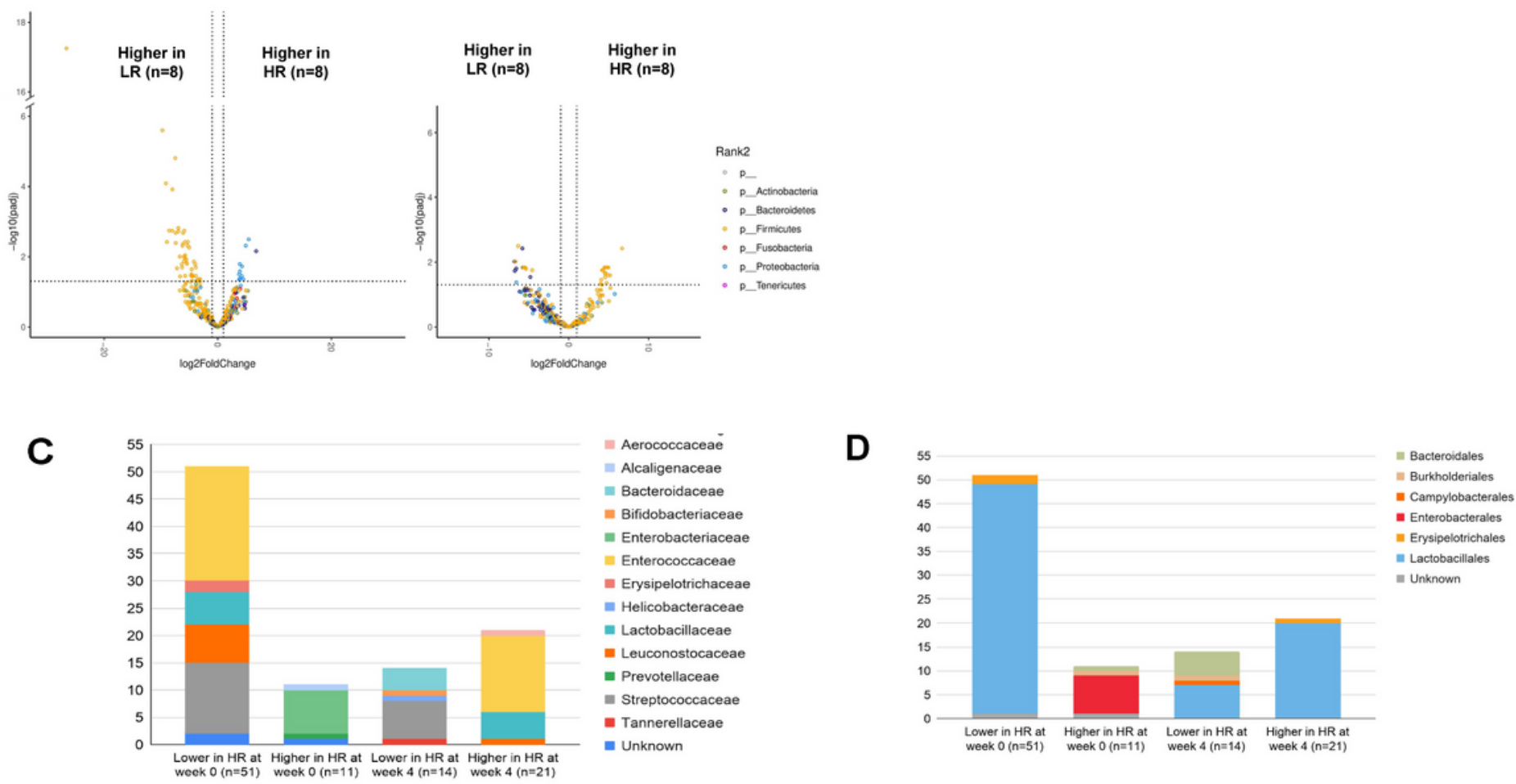

\section{Figure 6}

Volcano plots demonstrating fold-change $(\mathrm{FC})$ abundance of gut bacteria between HR (high-responders, $n=8$ ) and LR (low-responders, $n=8$ ) among dogs receiving the probiotics at (A) week 0 and (B) week 4. Numbers of species with significantly different abundances are shown at (C) the family level and (D) the order level.

\section{Supplementary Files}

This is a list of supplementary files associated with this preprint. Click to download.

- Table1.pdf

- Table2.pdf

- Table3.pdf

- Table4.pdf

- Table5.pdf

- Table6.pdf

- SupplementalTable1.pdf

- SupplementalTable2.pdf

- SupplementalTable3.pdf 
- SupplementalTable4.pdf

- SupplementalTable5.pdf 